\title{
Human Oocytes Express Both ACE2 and BSG Genes and Corresponding Proteins: Is SARS-CoV-2 Infection Possible?
}

\author{
Irma Virant-Klun ${ }^{1}$ (D) Franc Strle $^{1}$ \\ Accepted: 1 December 2020 / Published online: 5 January 2021 \\ (C) The Author(s), under exclusive licence to Springer Science+Business Media, LLC part of Springer Nature 2021
}

\begin{abstract}
In addition to a number of scientific and medical questions about SARS-CoV-2 infection that still need to be answered, there is also the question of how this highly virulent virus and COVID-19 disease affect gametogenesis in humans. Even more important is the question of whether the virus can also enter and infect oocytes and possibly alter them in an unknown way, which could also affect the development and status of the human embryo. The answers to these questions are still poorly known, so we reviewed the human oocyte transcriptome and proteome obtained in our previous studies and found that human oocytes from the in vitro fertilization program expressed both the $A C E 2$ and $B S G$ genes and the corresponding ACE2 and BSG proteins. This means that human oocytes possess the molecular 'machinery' to facilitate SARS-CoV-2 entrance and infection. According to various studies, especially in animal models, different viruses can infect oocytes, so infection of the oocyte with SARS-Cov-2 cannot be completely ruled out. A hypothetical model of human oocyte infection with this virus has been proposed.
\end{abstract}

Keywords SARS-CoV-2 $\cdot$ COVID-19 $\cdot$ Human $\cdot$ Oocyte $\cdot$ ACE2 $\cdot$ BSG

\section{Introduction}

SARS-CoV-2 is becoming our reality, as COVID-19 affects many people around the world. In a subset of patients, the disease is more severe and manifests with involvement of several organs. In addition to many scientific or medical questions that still need to be answered, there is also the question of how this virus and disease affect gametogenesis in humans. Even more important is the question of whether the virus can also infect and enter gametes and possibly alter them in an unknown way, which could also affect the development and status of the human embryo. The ability of SARS-CoV-2 to affect a tissue or cells is determined by its capacity to infect cells and replicate in them, which requires expression of genes and corresponding

This article belongs to the Topical Collection: Special Issue on COVID19 Pandemic and Stem Cells

Guest Editor: Mariusz Z. Ratajczak

Irma Virant-Klun

irma.virant@kclj.si

Franc Strle

franc.strle@kclj.si

1 Clinical Research Center, University Medical Center Ljubljana, Vrazov trg 1, Ljubljana, Slovenia proteins such as SARS-CoV-2 receptors angiotensin converting enzyme 2 (ACE2) and basigin (Ok Blood Group) (BSG = CD147), transmembrane serine protease 2 (TMPRSS2) and cathepsin L (CTSL) $[1,2]$. The mRNAs of all these genes are expressed in most of the human female reproductive tract, whole ovary [3] including follicular cumulus cells [4], and endometrium [5]. Genes ACE2 and MPRSS2 were expressed even in the human zygote and during the early developmental stages of the human embryo - the trophoblast of the preimplantation blastocyst, and syncytiotrophoblast and hypoblast of the implantation stages, which develop into tissues that interact with the maternal blood supply for nutrient exchange [6-8]. On the other hand, protein expression in these tissues has not been confirmed and is still unknown.

The organ- and cell-specific expression of these genes suggests that it may also play a role in the regulation of female fertility. In an animal model, the involvement of the ACE2 gene in the ovulation process has been demonstrated. This gene was expressed in follicular - theca and granulosa - cells and was affected by application of gonadotropin-releasing hormone $(\mathrm{GnRH})$ in granulosa cells [9]. The recent observation of proteomic data in HIPED revealed enrichment of ACE2 protein in the human placenta and ovary despite a low mRNA level [2]. One study also showed that the lack of expression of the $B S G$ gene which encodes a transmembrane glycoprotein belonging to the immunoglobulin in mice causes 
infertility, mainly due to defects in fertilization and implantation of embryos [10]. We can assume that the virus infection might affect the molecular events in the ovarian follicle, ovulation, fertilization and embryo implantation and consequently female fertility.

There is much less knowledge about oocytes in humans. An excellent model for analysis is represented by oocytes from the in vitro fertilization program, which are not fertilized by the in vitro fertilization procedure or are immature and do not have the natural ability to fertilize. It has recently been reported that human (primary) oocytes and preimplantation embryos (blastocystst) from the in vitro fertilization program express the ACE2 and BSG receptors as determined by immunocytochemistry [6]. At present, there is no data on expression of $A C E 2$ and $B S G$ genes and corresponding proteins in human oocytes, especially mature (secondary) oocytes. Therefore, we examined the human mature oocyte transcriptome [11] and proteome [12] obtained in our previous studies in which we did not focus on these two genes and proteins to determine whether human oocytes expressed the $A C E 2$ and $B S G$ genes and corresponding proteins. In other words, we were interested in whether human oocytes from the in vitro fertilization program have a molecular apparatus that allows infection with SARS-CoV-2.

\section{Expression of Genes ACE2 and BSG in Human Oocytes as Revealed by Transcriptomics}

Our transcriptome data [11] were obtained by microarray analysis (Agilent Whole Human Genome Oligo Microarray $8 \times 60 \mathrm{~K}$ v2 platform) of groups of human oocytes (12 groups of oocytes with 10 oocytes per group) after SuperAmp RNA amplification and showed that oocytes expressed both ACE2 and $B S G$ genes. The normalization was performed with multi lowess algorithm since it effectively removes systematic errors keeping a maximum of the original signal.

Interestingly, the gene $A C E 2$ was more expressed in oocytes that matured in vitro by a special laboratory procedure (maturation medium with added follicle-stimulating hormoneFSH and human chorionic gonadotropin-HCG, in coculture with cumulus cells from mature oocytes in the same women) than in oocytes that matured in vivo (in the ovaries) and were obtained as mature by ultrasound-guided aspiration of ovarian follicles for the procedure of in vitro fertilization. The ACE2 gene was among 25 top genes which were most differently expressed between oocytes matured in vitro and in vivo, as ordered by T-test fold comparing in vivo matured oocytes with the rest - in vitro matured oocytes ( 2.683 vs. 8.949 , adjusted T-Test $\mathrm{P}$ value $=0.0712$ ); its expression level was quite comparable to some important transcription factors such as general transcription factor GTF2H5 and RQCD1 (= CCR4-NOT Transcription Complex Subunit 9-CNOT9), which play an important role in the oocyte gene transcription regulation and DNA repair (2.683 vs. 3.893 and 2.414, respectively). In oocytes that matured in vivo, the $B S G$ gene was more expressed than the $A C E 2$ gene ( 8.935 vs. 2.6583). In oocytes matured in vivo, however, the expression of the $B S G$ gene was comparable to oocytes matured in vitro (8.935 vs. 9.301) with less than 2-fold difference in expression.

\section{Expression of Proteins ACE2 and BSG as Revealed by Proteomics}

Exploiting SP3, a novel technology for proteomic sample preparation using magnetic beads, the proteome was scaled down from 100 oocytes to single oocytes [12]. Samples containing 100, 10, 5 or single human oocytes from the in vitro fertilization program that did not fertilize after in vitro fertilization procedure were processed via SP3, a single-tube method utilizing magnetic beads for unbiased protein and peptide capture and recovery as described previously [13]. Proteins/peptides were analyzed by LC-MSMS (liquid chromatography attached to a mass spectrometer).

Upon examination of this first human oocyte proteome, we found that the oocytes expressed both the ACE and BSG protein. The protein ACE (soluble form) was identified only in groups of 100 oocytes thus indicating a relatively low expression of this protein in human oocytes. The protein BSG was identified in groups of 100,10 , and 5 oocytes, and even in single oocytes thus suggesting a relatively high expression of this protein in human oocytes in comparison to ACE. These proteins were expressed in both mature and immature oocytes. On the other hand, the ACE and BSG proteins were not identified in the oocyte secretome (proteins/peptides collected by overnight culturing oocytes in hanging drops of culture medium).

\section{Assessment of a Hypothetical Human Oocyte Infection with SARS-CoV-2}

Since human oocytes express functional receptors for the SARS-Cov-2 - both a gene and a protein for ACE2 and BSG - this virus could theoretically infect the oocytes. However, the prerequisite for the binding of a virus to receptor and entrance of the virus into the cell is the presence of the virus at the oocyte. There are two hypothetical ways for a virus (including SARS-CoV-2) to be delivered to human oocyte: blood-borne transmission or sexual transmission.

It is well established that the dominant mode of SARS-CoV2 transmission is respiratory transmission, with proximity and ventilation being crucial factors of transmission risk [14]. The information on blood-borne transmission or sexual transmission is much more limited than on respiratory transmission.

Current evidence does not support sexual transmission of SARS-CoV-2. Even though SARS-CoV-2 RNA has been found in semen, infectious virus has not been isolated [15] and vaginal fluid has been negative except in a single case 
that reported RNA with a low viral level $[16,17]$. Furthermore, there are no reported cases of SARS-CoV-2 transmission via sexual route [14].

According to a recent review on transmission of SARS-CoV2 , similar conclusions are also valid for blood-borne transmission [14] with a little bit more uncertainties. Findings on the proportion of persons with viral RNA detectable in blood are rather heterogeneous. For example, in one study [18] SARS-CoV-2 RNA was demonstrated in $1 \%$ (3/307) blood samples, in the other [19] in 33\% (28/85) blood specimens obtained from symptomatic persons (including 22/28, $79 \%$ of those requiring hospitalization), while in the another study SARS-CoV-2 RNA was established in blood in 27\% (19/71) of hospitalized patients and in 13\% (2/16) of outpatients with COVID-19 [20]. Furthermore, viral RNA was also found in blood from 4 blood donors without symptoms [21]. However, to date, no replication-competent virus has been isolated from blood samples, and there are no documented cases of blood-borne transmission of virus [14]. Yet, some studies suggest that vertical transmission of SARS-CoV-2 occurs, including several reports of positive SARS-CoV-2 IgM in neonates $[22,23]$ as well as a few reports of early nasopharyngeal positivity on polymerase chain reaction testing in neonates after delivery [24, 25]. In addition, several case reports on placental infection by SARS-CoV-2, including one on transplacental transmission, have been published [26-29].

Taken together, although human oocytes express functional receptors for the SARS-CoV-2, the ways for the virus to be delivered to human oocyte have not been reliably established. Currently, the assumption on the potential blood-borne transmission appears to be slightly more likely than sexual transmission.

\section{Potential SARS-CoV-2 Infection of the Human Oocyte at the Cellular Level}

The human oocyte could become infected inside the ovarian follicle (Fig. 1). In the microenvironment of the oocyte, the source of infection could be blood flowing through capillaries to the ovarian follicle. When infected in the natural condition, the virus should first infect the surrounding follicular cells inside the ovarian follicle - granulosa and theca cells - and then the oocyte via gap junctions and exudates of follicular cells. The virus could persist in the follicular fluid and infect the oocyte. In addition, the oocyte can also become infected during the fertilization process with infected spermatozoon. Under in vitro conditions, the oocyte could become infected during in vitro fertilization procedure either due to its handling (staff, material) or by the addition of potentially infected semen to fertilize it.

\section{Discussion}

We found that both $A C E 2$ and $B S G$ genes (transcripts) and corresponding proteins are expressed in human oocytes and, interestingly, the expression of gene $A C E 2$ was even higher in immature oocytes that matured in vitro by a lab procedure (clinically used in some in vitro fertilization laboratories) than in oocytes that matured in vivo.

Our observation is consistent with the study of Wang and co-workers who found the expression of ACE2 and BSG proteins in groups of mouse mature and immature oocytes (7000 oocytes per group) as well as zygotes [30]. The expression of ACE2 and BSG proteins in their study depended on oocyte maturity: ACE2 protein was expressed only in immature oocytes, while BSG protein was expressed in both immature and mature oocytes and zygotes. However, we did not find any other data for human oocytes in the literature.

It is clear from the literature that various viruses can infect the oocyte. The human hepatitis B virus (HBV) has already been detected in human oocytes and embryos from the in vitro fertilization program [31-33]. HBV mRNA was detected in the cleavage embryos of patients with chronic HBV infection, with a detection rate of $13.2 \%(5 / 38)$. It has also ben evidenced that oocytes of HBV carriers may express hepatitis B surface antigen (HBsAg) [34]. Viral antigens for herpes simplex virus was demonstrated in mature oocytes of infertile patients included in the in vitro fertilization program [35]. In general, various basic studies in animals (mammals) have shown that viruses can affect oocytes and, after fertilization, the embryo. Bovine oocytes infected with bovine diarrhea viruses BVDV-1 and BVDV-2, and Hobi-like virus resulted in embryos after in vitro fertilization [36]. Embryos from BVDV-1 and BVDV-2 infected oocytes developed normally, but carried the virus. However, Hobi-like virus infected oocytes had reduced cleavage potential and caused the preimplantation embryo loss, but viable embryos did not carry the virus. Structural changes in the zona pellucida (ZP) of bovine oocytes seem to modulate their interaction with various viral agents, facilitating the viral infection in in vitro production systems; the BFDV viruses were detected in granulosa cells, ZP-free mature oocytes but also ZP-intact mature oocytes [37]. It has been evidenced that bovine herpesvirus 1 can negatively impact the bovine oocyte development during in vitro maturation procedure [38]. Moreover, infection of porcine oocytes with porcine circovirus type 2 affected embryo quality after in vitro fertilization, more specifically the reduced numbers of cells in blastocysts [39]. All this data suggests that viruses can infect the mammalian oocyte and affect the development of the preimplantation embryo, so infection with the SARS-CoV-2 cannot be completely ruled out.

According to a review of the literature, it is possible that SARS-CoV-2 negatively affects the female fertility. The available evidence suggests that gene $A C E 2$ is widely expressed in the ovary, uterus, vagina and placenta [40]. Therefore, it is believed that apart from droplets and contact transmission, the possibility of mother-to-child and sexual transmission also exists. Along with other genes, the gene 


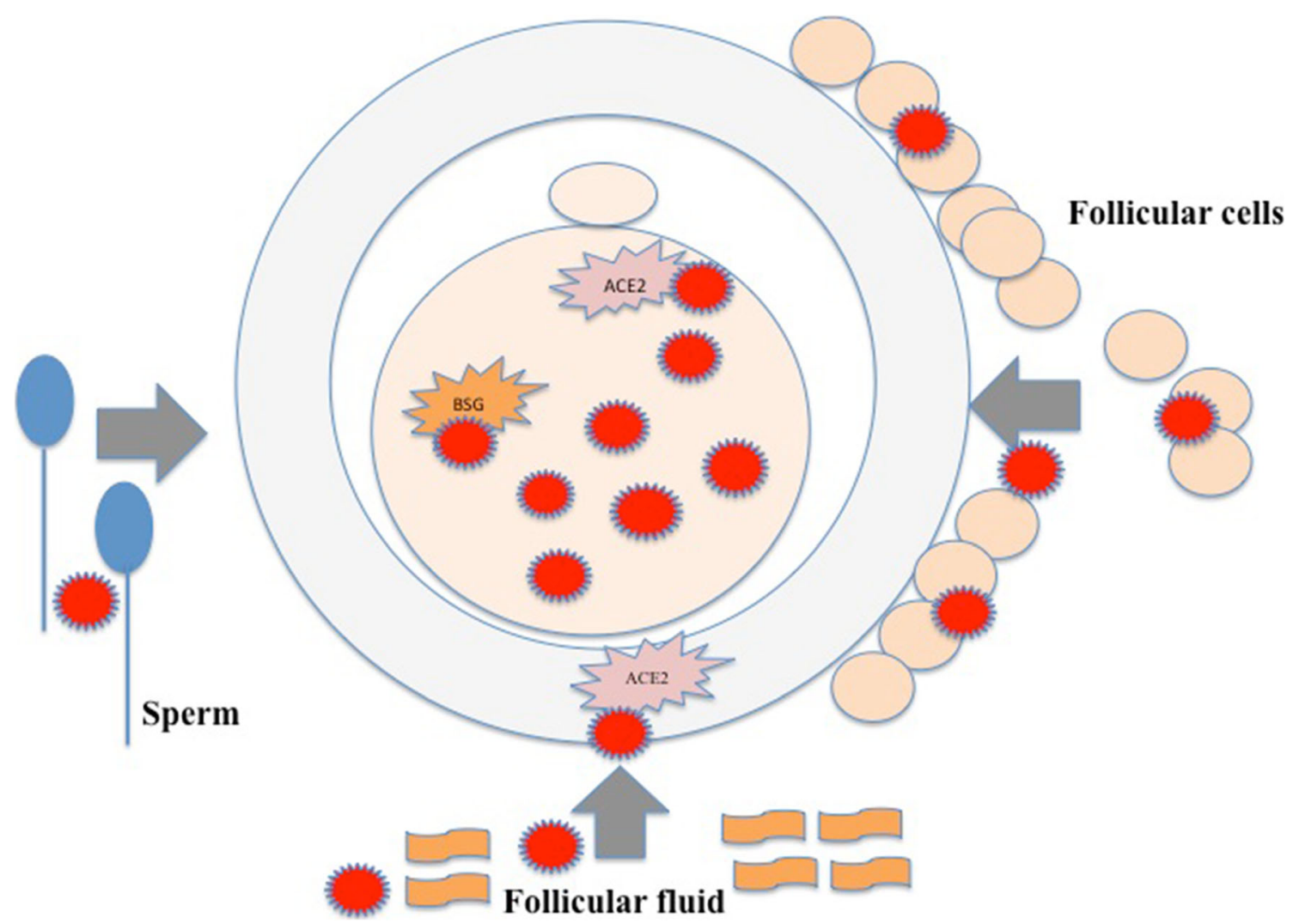

Fig. 1 Potential SARS-Cov-2 infection of the human oocyte at the cellular level via follicular cells and follicular fluid in the follicle and by sperm during the in vivo or in vitro fertilization process

$A C E 2$ regulates the ovarian follicle development and ovulation, modulate luteal angiogenesis and degeneration, and also influence the regular changes in endometrial tissue and embryo development [40]. In addition, $B S G$ gene expression has been shown to cause mouse infertility with an abnormal fertilization process and impaired embryo implantation [10]. Moreover, it was found that the concentration of BSG protein in the follicular fluid is associated with clinical pregnancy rate in patients undergoing in vitro fertilization, more specifically intracytoplasmic sperm injection (ICSI); at lower concentrations of this protein, the clinical pregnancy rate was higher, therefore, this protein was proposed as a marker for predicting ICSI outcomes [41]. Taking these functions into account, SARS-CoV-2 might disturb the female reproductive functions through regulating the $A C E 2$ and $B S G$ gene expression.

However, data on SARS-CoV-2 presence in human oocytes of infected individuals are poorly available to date. Recently, the first case of two women who underwent controlled ovarian stimulation for oocyte donation and tested positive to SARS-CoV-2 infection by PCR on the day of oocyte collection was reported [42]. The viral RNA for gene $\mathrm{N}$ was undetectable in all the oocytes analyzed from these two women. Nevertheless, no conclusion can be drawn as the number of oocytes analyzed (a total of 16 oocytes: 6 in one woman and 10 in second one) was small and only in two women [42]. In addition, the virus could have a negative effect on the oocytes in other ways (for example, with toxins, altered metabolism, changes within the ovarian follicle or ovarian niche).

It is worrying that the (potential) infection or other negative effects of SARS-CoV-2 on very small embryonic-like stem cells (VSELs), which are proposed as precursors of oocytes, pluripotent embryonic stem cells and organoids, and some other types of stem cells including hematopoietic stem cells has been proven [43-50]. Human embryonic stem cellderived organoids are proposed to be used as an efficient tool to study SARS-CoV-2 infection [51]. This also indicates a possible infection and negative effects of SARS-CoV-2 on the human oocyte and pre- and post-implantation embryo.

We may conclude that at present it is not clear whether the highly virulent SARS-CoV-2 can infect human oocytes, and whether the use of oocytes from women harboring the virus can result in an infection of the developing embryo in the in vitro fertilization program or in natural conditions. This important question needs to be answered in the future. Our transcriptomic and proteomic data on ACE2 and BSG expression in human oocytes show that this might be possible and that oocytes possess the molecular machinery to facilitate SARS-CoV-2 entrance. A hypothetical model of oocyte infection with this virus has been proposed. 
Acknowledgements We would like to thank Prof. Jeroen Krijgsveld, Head of Proteomics of Stem Cells and Cancer, German Cancer Research Center, Heidelberg, Germany and Prof. Thomas Skutella, Head of Research Unit, Institute for Anatomy and Cell Biology, Medical Faculty, University of Heidelberg, Germany for collaboration in previous experimental studies to gain a better insight into the molecular level of the human oocyte that resulted in the transcriptome and first proteome of human oocytes from in vitro fertilization program.

Author Contributions IVK Wrote the manuscript.

FS Wrote the section on Assessment of a hypothetical human oocyte infection with SARS-CoV-2 and corrected the manuscript.

Data Availability All data on the transcriptome and proteome of human oocytes are available at the Clinical Research Center, University Medical Center Ljubljana. They were also previously published and are available in cited publications.

\section{Compliance with Ethical Standards}

Conflict of Interest The authors declare that there is no conflict of interest.

Ethics Approval This work was approved by the Slovenian National Medical Ethical Committee (Ministry of Health RS; KME 23 k/10/12).

Consent to Participate Not applicable. This is a review manuscript. In previous studies, oocytes were included in the transcriptome and proteome analysis after the written informed consents of patients.

Consent for Publication The authors agree to publish this manuscript.

\section{References}

1. Hoffmann, M., Kleine-Weber, H., Schroeder, S., Kruger, N., Herrler, T., Erichsen, S., Schiergens, T. S., Herrler, G., Wu, N. H., Nitsche, A., Müller, M. A., Drosten, C., \& Pöhlmann, S. (2020). SARS-CoV-2 cell entry depends on ACE2 and TMPRSS2 and is blocked by a clinically proven protease inhibitor. Cell, 181(2), 271-280.

2. Wang, Y., Wang, Y., Luo, W., Huang, L., Xiao, J., Li, F., Qin, S., Song, X., Wu, Y., Zeng, Q., Jin, F., \& Wang, Y. (2020). A comprehensive investigation of the mRNA and protein level of ACE2, the putative receptor of SARS-CoV-2, in human tissues and blood cells. International Journal of Medical Sciences, 17(11), 15221531.

3. Hikmet, F., Mear, L., Edvinsson, A., Micke, P., Uhlen, M., \& Lindskog, C. (2020). The protein expression profile of ACE2 in human tissues. Molecular Systems Biology, 16(7), e9610.

4. Stanley, K. E., Thomas, E., Leaver, M., \& Wells, D. (2020). Coronavirus disease- 19 and fertility: viral host entry protein expression in male and female reproductive tissues. Fertility \& Sterility, 114(1), 33-43.

5. Henarejos-Castillo, I., Sebastian-Leon, P., Devesa-Peiro, A., Pellicer, A., \& Diaz-Gimeno, P. (2020). SARS-CoV-2 infection risk assessment in the endometrium: viral infection-related gene expression across the menstrual cycle. Fertility \& Sterility, 114(2), 223-232.

6. Essahib, W., Verheyen, G., Tournaye, H., \& Van de Velde, H. (2020). SARS-CoV-2 host receptors ACE2 and CD147 (BSG) are present on human oocytes and blastocysts. Journal of Assisted Reproduction and Genetics, 37(11), 2657-2660.
7. Weatherbee, B. A. T., Glover, D. M., \& Zernicka-Goetz, M. (2020). Expression of SARS-CoV-2 receptor ACE2 and the protease TMPRSS2 suggests susceptibility of the human embryo in the first trimester. Open Biology, 10(8), 200162.

8. Stirparo, G. G., Boroviak, T., Guo, G., Nichols, J., Smith, A., \& Bertone, P. (2018). Integrated analysis of single-cell embryo data yields a unified transcriptome signature for the human preimplantation epiblast. Development, 145(3), dev158501.

9. Tonellotto dos Santos, J., Ferreira, R., Gasperin, B. G., Siqueira, L. C., de Oliveira, J. F., Santos, R. A., Reis, A. M., \& Gonçalves, P. B. (2012). Molecular characterization and regulation of the angiotensin-converting enzyme type 2/angiotensin-(1-7)/MAS receptor axis during the ovulation process in cattle. Journal of the Renin-Angiotensin-Aldosterone System, 13(1), 91-98.

10. Kuno, N., Kadomatsu, K., Fan, Q. W., Hagihara, M., Senda, T., Mizutani, S., \& Muramatsu, T. (1998). Female sterility in mice lacking the basigin gene, which encodes a transmembrane glycoprotein belonging to the immunoglobulin superfamily. FEBS Letters, 425(2), 191-194.

11. Virant-Klun, I., Bauer, C., Ståhlberg, A., Kubista, M., \& Skutella, T. (2018). Human oocyte maturation in vitro is improved by coculture with cumulus cells from mature oocytes. Reproductive Biomedicine Online, 36(5), 508-523.

12. Virant-Klun, I., Leicht, S., Hughes, C., \& Krijgsveld, J. (2016). Identification of maturation-specific proteins by single-cell proteomics of human oocytes. Molecular \& Cellular Proteomics, 15(8), 2616-2627.

13. Hughes, C. S., Foehr, S., Garfield, D. A., Furlong, E. E., Steinmetz, L. M., \& Krijgsveld, J. (2014). Ultrasensitive proteome analysis using paramagnetic bead technology. Molecular Systems Biology, 10(10), 757

14. Meyerowitz, E. A., Richterman, A., Gandhi, R. T., \& Sax, P. E. (2020). Transmission of SARS-CoV-2: A review of viral, host, and environmental factors. Annals of Internal Medicine. https://doi.org/ 10.7326/M20-5008.

15. Li, D., Jin, M., Bao, P., Zhao, W., \& Zhang, S. (2020). Clinical characteristics and results of semen tests among men with coronavirus disease 2019. JAMA Network Open, 3(5), e208292.

16. Qiu, L., Liu, X., Xiao, M., Xie, J., Cao, W., Liu, Z., Morse, A., Xie, Y., Li, T., \& Zhu, L. (2020). SARS-CoV-2 is not detectable in the vaginal fluid of women with severe COVID-19 infection. Clinical Infectious Diseases, 71(15), 813-817.

17. Scorzolini, L., Corpolongo, A., Castilletti, C., Lalle, E., Mariano, A., \& Nicastri, E. (2020). Comment of the potential risks of sexual and vertical transmission of Covid-19 infection. Clinical Infectious Diseases, 71(16), 2298.

18. Wang, W., Xu, Y., Gao, R., Lu, R., Han, K., Wu, G., \& Tan, W. (2020). Detection of SARS-CoV-2 in different types of clinical specimens. JAMA, 323(18), 1843-1844.

19. Hogan, C. A., Stevens, B., Sahoo, M. K., Huang, C. H., Garamani, N., Gombar, S., Yamamoto, F., Murugesan, K., Kurzer, J., Zehnder, J., \& Pinsky, B. A. (2020). High frequency of SARS-CoV-2 RNAemia and association with severe disease. Clinical Infectious Diseases. doi: https://doi.org/10.1101/2020.04.26.20080101.

20. Fajnzylber, J. M., Regan, J., Coxen, K., Corry, H., Wong, C., Rosenthal, A., Worrall, D., Giguel, F., Piechocka-Trocha, A., Atyeo, C., Fischinger, S., Chan, A., Flaherty, K. T., Hall, K., Dougan, M., Ryan, E. T., Gillespie, E., Chishti, R., Li, Y., Jilg, N., Hanidziar, D., Baron, R. M., Baden, L., Tsibris, M., Armstrong, K. A., Kuritzkes, D. R., Alter, G., Walker, B. D., Yu, X., \& Li, J. (2020). SARS-CoV-2 viral load is associated with increased disease severity and mortality. MedRxiv. https://doi.org/ 10.1101/2020.07.15.20131789.

21. Chang, L., Zhao, L., Gong, H., Wang, L., \& Wang, L. (2020). Severe acute respiratory syndrome coronavirus 2 RNA detected in blood donations. Emerging Infectious Diseases, 26(7), 1631-1633. 
22. Zeng, H., Xu, C., Fan, J., Tang, Y., Deng, Q., Zhang, W., \& Long, X. (2020). Antibodies in infants born to mothers with COVID-19 pneumonia. JAMA, 323(18), 1848-1849.

23. Dong, L., Tian, J., He, S., Zhu, C., Wang, J., Liu, C., \& Yang, J. (2020). Possible vertical transmission of SARS-CoV-2 from an infected mother to her newborn. JAMA, 323(18), 1846-1848.

24. Zeng, L., Xia, S., Yuan, W., Yan, K., Xiao, F., Shao, J., \& Zhou, W. (2020). Neonatal early-onset infection with SARS-CoV-2 in 33 neonates born to mothers with COVID-19 in Wuhan, China. JAMA Pediatrics, 174(7), 722-725.

25. Alzamora, M. C., Paredes, T., Caceres, D., Webb, C. M., Valdez, L. M., \& La Rosa, M. (2020). Severe COVID-19 during pregnancy and possible vertical transmission. American Journal of Perinatology, 37(8), 861-865.

26. Patanè, L., Morotti, D., Giunta, M. R., Sigismondi, C., Piccoli, M. G., Frigerio, L., Mangili, G., Arosio, M., \& Cornolti, G. (2020). Vertical transmission of coronavirus disease 2019: severe acute respiratory syndrome coronavirus 2 RNA on the fetal side of the placenta in pregnancies with coronavirus disease 2019-positive mothers and neonates at birth. American Journal of Obstetrics \& Gynecology MFM, 2(3), 100145.

27. Baud, D., Greub, G., Favre, G., Gengler, C., Jaton, K., Dubruc, E., \& Pomar, L. (2020). Second-trimester miscarriage in a pregnant woman with SARS-CoV-2 infection. JAMA, 323(21), 2198-2200.

28. Vivanti, A. J., Vauloup-Fellous, C., Prevot, S., Zupan, V., Suffee, C., Cao, D., Benachi, J. A., \& De Luca, D. (2020). Transplacental transmission of SARS-CoV-2 infection. Nature Communications, 11(1), 3572 .

29. Alamar, I., Abu-Arja, M. H., Heyman, T., Roberts, D. J., Desai, N., Narula, P., \& Dygulska, B. (2020). A possible case of vertical transmission of SARS-CoV-2 in a newborn with positive placental in situ hybridization of SARS-CoV-2 RNA. Journal of the Pediatric Infectious Diseases Society, 9(5), 636-639.

30. Wang, S., Kou, Z., Jing, Z., Zhang, Y., Guo, X., Dong, M., Wilmut, I., \& Gao, S. (2010). Proteome of mouse oocytes at different developmental stages. Proceedings of the National Academy of Sciences of the United States of America, 107(41), 17639-17644.

31. Ghosh, M., Nandi, S., Dutta, S., \& Saha, M. K. (2015). Detection of hepatitis B virus infection: A systematic review. World Journal of Hepatology, 7(23), 2482-2491.

32. Ye, F., Jin, Y., Kong, Y., Shi, J. Z., Qiu, H. T., Zhang, X., Zhang, S. L., \& Lin, S. M. (2013). The presence of HBV mRNA in the fertilized in vitro embryo of HBV patients confirms vertical transmission of HBV via the ovum. Epidemiology \& Infection, 141(5), 926-930.

33. Nie, R., Jin, L., Zhang, H., Xu, B., Chen, W., \& Zhu, G. (2011). Presence of hepatitis $\mathrm{B}$ virus in oocytes and embryos: a risk of hepatitis B virus transmission during in vitro fertilization. Fertility \& Sterility, 95(5), 1667-1671.

34. Lou, H., Ding, W., Dong, M., Zhu, Y., Zhou, C., Wang, Z., Yang, X., Yao, Q., Li, D., \& Miao, M. (2010). The presence of hepatitis B surface antigen in the ova of pregnant women and its relationship with intra-uterine infection by hepatitis B virus. Journal of International Medical Research, 38(1), 214-219.

35. Abdulmedzhidova, A. G., Rog, K. V., Zavalishina, L., \& Kushch, A. A. (2014). Intrafollicular infection of mammals and human oocytes by the herpes simplex virus. Voprosy Virusologii, 59(1), 4246.

36. Da Silva Cardoso Pinto, V., Alves, M. F., de Souza Nunes Martins, M., Basso, A. C., Tannura, J. H., Pontes, J. H. F., Lima, M. S., Garcia da Silva, T., Okuda, L. H., Stefano, E., Romaldini, A. H. C. N., Arnold, D. R., \& Pituco, E. M. (2017). Effects of oocytes exposure to bovine diarrhea viruses BVDV-1, BVDV-2 and Hobilike virus on in vitro-produced bovine embryo development and viral infection. Theriogenology, 97, 67-72.
37. González Altamiranda, E. A., Kaiser, G. G., Ríos, G. L., Leunda, M. R., \& Odeón, A. C. (2016). Interaction of bovine viral diarrhea virus with bovine cumulus-oocyte complex during IVM: Detection in permissive cells. Theriogenology, 86(8), 1999-2003.

38. Alves, S. V. P., da Costa, E. P., Queiroz-Castro, V. L. D., MachadoNeves, M., Guimarães, J. D., Gomes, L. L., Junior, A. M. A., \& Júnior, A. S. (2019). Bovine herpesvirus 1 can impact the bovine oocyte development during in vitro maturation. Research in Veterinary Science, 123, 135-140.

39. Weng, X. G., Liu, Y., Zhou, S. H., Zhang, Y. T., Shao, Y. T., Xu, Q. Q., \& Liu, Z. H. (2019). Evaluation of porcine circovirus type 2 infection in in vitro embryo production using naturally infected oocytes. Theriogenology, 126, 75-80.

40. Jing, Y., Run-Qian, L., Hao-Ran, W., Hao-Ran, C., Ya-Bin, L., Yang, G., \& Fei, C. (2020). Potential influence of COVID-19/ ACE2 on the female reproductive system. Molecular Human Reproduction, 26(6), 367-373.

41. Takmaz, O., Yozgatli, D., Ozaltin, S., Ozbasli, E., Kocyigit, Y., Kuran, S. B., Bulut, H., Gungor, M., Buyru, F., \& Bastu, E. (2019). Can follicular Emmprin and BMP 4 levels predict ICSI outcome? Assisted Reproduction and Genetics, 36(6), 1127-1133.

42. Barragan, M., Guillén, J. J., Martin-Palomino, N., Rodriguez, A., \& Vassena, R. (2020). Undetectable viral RNA in oocytes from SARS-CoV-2 positive women. Human Reproduction. doi:https:// doi.org/10.1093/humrep/deaa284.

43. Ratajczak, M. Z., Bujko, K., Ciechanowicz, A., Sielatycka, K., Cymer, M., Marlicz, W., \& Kucia, M. (2020). SARS-CoV-2 entry receptor ACE2 is expressed on very small CD45- precursors of hematopoietic and endothelial cells and in response to virus spike protein activates the Nlrp3 inflammasome. Stem Cell Reviews and Reports. https://doi.org/10.1007/s12015-020-10010-z.

44. Huang, J., Hume, A. J., Abo, K. M., Werder, R. B., VillacortaMartin, C., Alysandratos, K. D., Beermann, M. L., SimoneRoach, C., Olejnik, Lindstrom-VautrinJ., Suder, J., Bullitt, E. L., Hinds, E., Sharma, A., Bosmann, A., Wang, M., Hawkins, R., Burks, F., Saeed, E. J., Wilson, M., Mühlberger, A. A., E., \& Kotton, D. N. (2020). SARS-CoV-2 infection of pluripotent stem cell-derived human lung alveolar type 2 cells elicits a rapid epithelial-intrinsic inflammatory response. bioRxiv. https://doi.org/ 10.1101/2020.06.30.175695.

45. Makovoz, B., Moeller, R., Zebitz Eriksen, A., tenOever, B. R., \& Blenkinsop, T. A. (2020). SARS-CoV-2 infection of ocular cells from human adult donor eyes and hESC-derived eye organoids. SSRN. https://doi.org/10.2139/ssrn.3650574.

46. Zhang, B. Z., Chu, H., Han, S., Shuai, H., Deng, J., Hu, Y. F., Gong, H. R., Lee, A. C., Zou, Z., Yau, T., Wu, W., Hung, I. F., Chan, J. F., Yuen, K. Y., \& Huang, J. D. (2020). SARS-CoV-2 infects human neural progenitor cells and brain organoids. Cell Research, 30(10), 928-931.

47. Yi, S. A., Nam, K. H., Yun, J., Gim, D., Joe, D., Kim, Y. H., Kim, H. J., Han, J. W., \& Lee J. (2020). Infection of brain organoids and 2D cortical neurons with SARS-CoV-2 pseudovirus. Viruses, 12(9), 1004

48. Valyaeva, A. A., Zharikova, A. A., Kasianov, A. S., Vassetzky, Y. S., \& Sheval, E. V. (2020). Expression of SARS-CoV-2 entry factors in lung epithelial stem cells and its potential implications for COVID-19. Scientific Reports, 10(1), 17772.

49. Ropa, J., Cooper, S., Capitano, M. L., Van't Hof, W., \& Broxmeyer, H. E. (2020). Human hematopoietic stem, progenitor, and immune cells respond Ex Vivo to SARS-CoV-2 spike protein. Stem Cell Reviews and Reports. https://doi.org/10.1007/s12015020-10056-z.

50. Ratajczak, M. Z., \& Kucia, M. (2020). SARS-CoV-2 infection and overactivation of Nlrp3 inflammasome as a trigger of cytokine "storm" and risk factor for damage of hematopoietic stem cells. Leukemia, 34(7), 1726-1729. 
51. Salahudeen, A. A., Choi, S. S., Rustagi, A., Zhu, J., de la O, S. M., Flynn, R. A., Margalef-Català, M., Santos, A. J. M., Ju, J., Batish, A., van Unen, V., Usui, T., Zheng, G. X. Y., Edwards, C. E., Wagar, L. E., Luca, V., Anchang, B., Nagendran, M., Nguyen, K., Hart, D. J., Terry, J. M., Belgrader, P., Ziraldo, S. B., Mikkelsen, T. S., Harbury, P. B., Glenn, J. S., Garcia, K. C., Davis, M. M., Baric, R. S., Sabatti, C., Amieva, M. R., Blish, C.
A., Desai, T. J., \& Kuo, C. J. (2020). Progenitor identification and SARS-CoV-2 infection in long-term human distal lung organoid cultures. bioRxiv. https://doi.org/10.1101/2020.07.27.212076.

Publisher's Note Springer Nature remains neutral with regard to jurisdictional claims in published maps and institutional affiliations. 